\title{
Factorization of exclusive electron-induced two-nucleon knockout
}

\author{
Camille Colle, ${ }^{*}$ Wim Cosyn, ${ }^{\dagger}$ Jan Ryckebusch, ${ }^{\ddagger}$ and Maarten Vanhalst ${ }^{\S}$ \\ Department of Physics and Astronomy, Ghent University, Proeftuinstraat 86, B-9000 Gent, Belgium
}

(Received 13 November 2013; published 10 February 2014)

\begin{abstract}
We investigate the factorization properties of the exclusive electroinduced two-nucleon knockout reaction $A\left(e, e^{\prime} p N\right)$. A factorized expression for the cross section is derived and the conditions for factorization are studied. The $A\left(e, e^{\prime} p N\right)$ cross section is shown to be proportional to the conditional center-of-mass (c.m.) momentum distribution for close-proximity pairs in a state with zero relative orbital momentum and zero radial quantum number. The width of this conditional c.m. momentum distribution is larger than the one corresponding with the full c.m. momentum distribution. It is shown that the final-state interactions (FSIs) only moderately affect the shape of the factorization function for the $A\left(e, e^{\prime} p N\right)$ cross sections. Another prediction of the proposed factorization is that the mass dependence of the $A\left(e, e^{\prime} p p\right)\left[A\left(e, e^{\prime} p n\right)\right]$ cross sections is much softer than $\frac{Z(Z-1)}{2}[N Z]$.

DOI: 10.1103/PhysRevC.89.024603

PACS number(s): 25.30.Rw, 25.30.Fj, 24.10.-i
\end{abstract}

\section{INTRODUCTION}

In recent years, substantial progress has been made in exploring the dynamics of short-range correlations (SRCs) in nuclei. On the experimental side, exclusive $A(p, 2 p+n)$ [1] and $A\left(e, e^{\prime} p N\right)[2-4]$ measurements have probed correlated pairs in nuclei and identified proton-neutron $(p n)$ pairs as the dominant contribution. Inclusive $A\left(e, e^{\prime}\right)$ [5-7] measurements in kinematics favoring correlated pair knockout, have provided access to the mass dependence of the amount of correlated pairs relative to the deuteron. On the theoretical side, ab initio [8-11], cluster expansion [12-14], correlated basis function theory $[15,16]$, and low-momentum effective theory [17], calculations have provided insight in the fat highmomentum tails of the momentum distributions attributable to multinucleon correlations. Tensor correlations have been identified as the driving mechanism for the fat tails just above the Fermi momentum. The highest momenta in the tail of the momentum distribution are associated with the short-distance repulsive part of the nucleon-nucleon force and $N \geqslant 3$ correlations. Recent reviews of nuclear SRC can be found in Refs. [18,19].

We have proposed a method to quantify the amount of correlated pairs in an arbitrary nucleus [20-22]. Thereby, we start from a picture of a correlated nuclear wave function as a product of a correlation operator acting on an independentparticle model (IPM) Slater determinant $\Psi_{A}^{\mathrm{IPM}}$ [17]. The SRC-susceptible pairs are identified by selecting those parts of $\Psi_{A}^{\text {IPM }}$ that provide the largest contribution when subjected to typical nuclear correlation operators. It is found that IPM nucleon-nucleon pairs with vanishing relative orbital momentum and vanishing relative radial quantum numbers, receive the largest corrections from the correlation operators. This can be readily understood by realizing that IPM closeproximity pairs are highly susceptible to SRC corrections. This

\footnotetext{
*camille.colle@ugent.be

†wim.cosyn@ugent.be

${ }^{\ddagger}$ jan.ryckebusch@ugent.be

${ }^{\S}$ maarten.vanhalst@ugent.be
}

imposes constraints on the relative orbital and radial quantum numbers of the two-nucleon cluster components in the IPM wave functions which receive SRC corrections.

With the proposed method of quantifying SRC we can reasonably account for the mass dependence of the $\frac{A\left(e, e^{\prime}\right)}{d\left(e, e^{\prime}\right)}$ ratio under conditions of suppressed one-body contributions (Bjorken $x_{B} \gtrsim 1.2$ ) [21] and the mass dependence of the magnitude of the EMC effect $[22,23]$. In connecting the SRC information to inclusive electron-scattering data at Bjorken $x_{B} \gtrsim 1.2$, there are complicating issues like the role of c.m. motion [21,24] and final-state interactions (FSIs) [25]. More quantitative information on SRC and their mass and isospin dependence, is expected to come from exclusive electroinduced two-nucleon knockout which is the real fingerprint of nuclear SRC [26]. Reactions of this type are under investigation at Jefferson Laboratory (JLab) and results for ${ }^{12} \mathrm{C}\left(e, e^{\prime} p N\right)$ have been published $[3,4]$.

In this paper, we investigate the factorization properties of the exclusive $A\left(e, e^{\prime} p N\right)$ reaction. Factorization is a particular result that emerges only under specific assumptions in the description of the scattering process. It results in an approximate expression for the cross section which becomes proportional to a specific function of selected dynamic variables. For exclusive quasielastic $A\left(e, e^{\prime} p\right)$ processes, for example, the factorization function is the one-nucleon momentum distribution evaluated at the initial nucleon's momentum. It will be shown that for exclusive $A\left(e, e^{\prime} p N\right)$ these roles are respectively played by the c.m. momentum distribution for close-proximity pairs and the c.m. momentum of the initial pair.

In Sec. II we present calculations for the pair c.m. momentum distribution in the IPM. It is shown that the correlationsusceptible IPM pairs have a broader c.m. width than those that are less prone to SRC corrections. In Sec. III, we show that after making a number of reasonable assumptions, the eightfold $A\left(e, e^{\prime} p N\right)$ cross section factorizes with the conditional pair c.m. momentum distribution as the factorization function. In Sec. IV we report on results of Monte Carlo simulations for $A\left(e, e^{\prime} p p\right)$ processes in kinematics corresponding to those accessible in the JLab Hall A and Hall B detectors. We study the effect of typically applied cuts on several quantities. 
In Sec. V it is investigated to what extent FSIs affect the factorization function of the exclusive $A\left(e, e^{\prime} p N\right)$ process. Finally, our conclusions are stated in Sec. VI.

\section{PAIR CENTER-OF-MASS MOMENTUM DISTRIBUTIONS}

In this section we study the $p p$ and $p n$ pair c.m. momentum distribution for ${ }^{12} \mathrm{C},{ }^{27} \mathrm{Al},{ }^{56} \mathrm{Fe}$, and ${ }^{208} \mathrm{~Pb}$ which we deem representative for the full mass range of stable nuclei. We introduce the relative and c.m. coordinates and momenta

$$
\begin{array}{ll}
\vec{r}_{12}=\vec{r}_{1}-\vec{r}_{2}, & \vec{R}_{12}=\frac{\vec{r}_{1}+\vec{r}_{2}}{2}, \\
\vec{k}_{12}=\frac{\vec{k}_{1}-\vec{k}_{2}}{2}, & \vec{P}_{12}=\vec{k}_{1}+\vec{k}_{2} .
\end{array}
$$

The corresponding two-body momentum density reads

$$
\begin{aligned}
P_{2}\left(\vec{k}_{12}, \vec{P}_{12}\right)= & \frac{1}{(2 \pi)^{6}} \int d \vec{r}_{12} \int d \vec{r}_{12}^{\prime} \int d \vec{R}_{12} \int d \vec{R}_{12}^{\prime} \\
& \times e^{l \vec{k}_{12} \cdot\left(\vec{r}_{12}^{\prime}-\vec{r}_{12}\right)} e^{l \vec{P}_{12} \cdot\left(\vec{R}_{12}^{\prime}-\vec{R}_{12}\right)} \rho_{2}\left(\vec{r}_{12}^{\prime}, \vec{R}_{12}^{\prime} ; \vec{r}_{12}, \vec{R}_{12}\right),
\end{aligned}
$$

where $\rho_{2}\left(\vec{r}_{12}^{\prime}, \vec{R}_{12}^{\prime} ; \vec{r}_{12}, \vec{R}_{12}\right)$ is the nondiagonal two-body density (TBD) matrix

$$
\begin{aligned}
& \rho_{2}\left(\vec{r}_{12}^{\prime}, \vec{R}_{12}^{\prime}, \vec{r}_{12}, \vec{R}_{12}\right) \\
& \quad=\int\left\{d \vec{r}_{3-A}\right\} \Psi_{A}^{*}\left(\vec{r}_{1}^{\prime}, \vec{r}_{2}^{\prime}, \vec{r}_{3}, \ldots, \vec{r}_{A}\right) \Psi_{A}\left(\vec{r}_{1}, \vec{r}_{2}, \vec{r}_{3}, \ldots, \vec{r}_{A}\right) .
\end{aligned}
$$

Here, $\Psi_{A}$ is the normalized ground-state wave function of the nucleus $A$ and $\left\{d \vec{r}_{i-A}\right\} \equiv d \vec{r}_{i} d \vec{r}_{i+1}, \ldots, d \vec{r}_{A}$. For a spherically symmetric system, $P_{2}\left(\vec{k}_{12}, \vec{P}_{12}\right)$ depends on three independent variables, for example the magnitudes $\left|\vec{k}_{12}\right|$ and $\left|\vec{P}_{12}\right|$ and the angle between $\vec{k}_{12}$ and $\vec{P}_{12}$. In Ref. [13] two-body momentum distributions for ${ }^{3} \mathrm{He}$ and ${ }^{4} \mathrm{He}$ are shown to be largely independent of the angle between $\vec{k}_{12}$ and $\vec{P}_{12}$ for $P_{12} \lesssim 200 \mathrm{MeV}$. Integrating over the directional dependence of Eq. (3), the quantity

$$
\begin{aligned}
& n_{2}\left(k_{12}, P_{12}\right) k_{12}^{2} d k_{12} P_{12}^{2} d P_{12} \\
& \quad=k_{12}^{2} d k_{12} P_{12}^{2} d P_{12} \int d \Omega_{k_{12}} \int d \Omega_{P_{12}} P_{2}\left(\vec{k}_{12}, \vec{P}_{12}\right)
\end{aligned}
$$

is connected to the probability of finding a nucleon pair with relative and c.m. momentum in $\left[k_{12}, k_{12}+d k_{12}\right]$ and $\left[P_{12}, P_{12}+d P_{12}\right]$. With the spherical-wave expansion for the two vector plane waves in Eq. (3) one obtains

$$
n_{2}\left(k_{12}, P_{12}\right)=\frac{4}{\pi^{2}} \sum_{l m_{l}} \sum_{\Lambda M_{\Lambda}} n_{2}^{l m_{l} \Lambda M_{\Lambda}}\left(k_{12}, P_{12}\right),
$$

with

$$
\begin{aligned}
& n_{2}^{l m_{l} \Lambda M_{\Lambda}}\left(k_{12}, P_{12}\right) \\
& =\int d r_{12}^{\prime} r_{12}^{\prime 2} \int d R_{12}^{\prime} R_{12}^{\prime 2} \int d r_{12} r_{12}^{2} \int d R_{12} R_{12}^{2} \\
& \quad \times j_{l}\left(k_{12} r_{12}\right) j_{l}\left(k_{12} r_{12}^{\prime}\right) j_{\Lambda}\left(P_{12} R_{12}\right) j_{\Lambda}\left(P_{12} R_{12}^{\prime}\right) \\
& \quad \times \rho_{2}^{l m_{l} \Lambda M_{\Lambda}}\left(r_{12}^{\prime}, R_{12}^{\prime} ; r_{12}, R_{12}\right) .
\end{aligned}
$$

Here, $\rho_{2}^{l m_{l} \Lambda M_{\Lambda}}\left(r_{12}^{\prime}, R_{12}^{\prime} ; r_{12}, R_{12}\right)$ is the projection of the TBD matrix on relative and c.m. orbital angular-momentum states $\left|l m_{l}\right\rangle$ and $\left|\Lambda M_{\Lambda}\right\rangle$.

The pair c.m. momentum distribution is defined by

$$
\begin{aligned}
P_{2}\left(P_{12}\right) & =\int d \Omega_{P_{12}} \int d \vec{k}_{12} P_{2}\left(\vec{k}_{12}, \vec{P}_{12}\right) \\
& =\int d k_{12} k_{12}^{2} n_{2}\left(k_{12}, P_{12}\right),
\end{aligned}
$$

and the quantity $P_{2}\left(P_{12}\right) P_{12}^{2} d P_{12}$ is related to the probability of finding a nucleon pair with $\left|\vec{P}_{12}\right|$ in $\left[P_{12}, P_{12}+d P_{12}\right]$ irrespective of the magnitude and direction of $\vec{k}_{12}$. Similarly, the pair relative momentum distribution is defined as

$$
n_{2}\left(k_{12}\right)=\int d \Omega_{k_{12}} \int d \vec{P}_{12} P_{2}\left(\vec{k}_{12}, \vec{P}_{12}\right) .
$$

In the IPM, the ground-state wave function can be expanded in terms of single-particle wave functions $\phi_{\alpha_{i}}$

$$
\Psi_{A}^{\mathrm{IPM}}=(A !)^{-1 / 2} \operatorname{det}\left[\phi_{\alpha_{i}}\left(\vec{x}_{j}\right)\right],
$$

and the TBD matrix is given by

$$
\begin{aligned}
\rho_{2}^{\mathrm{IPM}} & \left(\vec{r}_{12}^{\prime}, \vec{R}_{12}^{\prime} ; \vec{r}_{12}, \vec{R}_{12}\right) \\
= & \frac{2}{A(A-1)} \sum_{\alpha<\beta} \frac{1}{2}\left[\phi_{\alpha}^{*}\left(\vec{x}_{1}^{\prime}\right) \phi_{\beta}^{*}\left(\vec{x}_{2}^{\prime}\right)-\phi_{\beta}^{*}\left(\vec{x}_{1}^{\prime}\right) \phi_{\alpha}^{*}\left(\vec{x}_{2}^{\prime}\right)\right] \\
& \times\left[\phi_{\alpha}\left(\vec{x}_{1}\right) \phi_{\beta}\left(\vec{x}_{2}\right)-\phi_{\beta}\left(\vec{x}_{1}\right) \phi_{\alpha}\left(\vec{x}_{2}\right)\right] .
\end{aligned}
$$

Here, $\vec{x} \equiv(\vec{r}, \vec{\sigma}, \vec{\tau})$ is a shorthand notation for the spatial, spin, and isospin coordinates. The summation $\sum_{\alpha<\beta}$ extends over all occupied single-particle levels and implicitly includes an integration over the spin and isospin degrees of freedom (d.o.f.).

In a $\mathrm{HO}$ basis the uncoupled single-particle states read

$$
\phi_{\alpha}(\vec{x}) \equiv \psi_{n_{\alpha} l_{\alpha} m_{l_{\alpha}}}(\vec{r}) \chi_{\sigma_{\alpha}}(\vec{\sigma}) \xi_{\tau_{\alpha}}(\vec{\tau}) .
$$

The $A$ dependence can be taken care of by means of the parametrization $\hbar \omega(\mathrm{MeV})=45 A^{\frac{1}{3}}-25 A^{\frac{2}{3}}$. A transformation from $\left(\vec{r}_{1}, \vec{r}_{2}\right)$ to $\left(\vec{r}_{12}, \vec{R}_{12}\right)$ for the uncoupled normalizedand-antisymmetrized (nas) two-nucleon states can be readily performed in a HO basis [20,21]

$$
|\alpha \beta\rangle_{\mathrm{nas}}=\sum_{\substack{n l m_{l} N \Lambda M_{\Lambda} \\ S M_{S} T M_{T}}}\left\langle n l m_{l} N \Lambda M_{\Lambda} S M_{S} T M_{T} \mid \alpha \beta\right\rangle\left|n l m_{l} N \Lambda M_{\Lambda} S M_{S} T M_{T}\right\rangle=\sum_{\substack{A=\left\{n l m_{l} N \Lambda M_{\Lambda} \\ S M_{S} T M_{T}\right\}}} C_{\alpha \beta}^{A}|A\rangle,
$$


with the transformation coefficient $C_{\alpha \beta}^{n l m m_{l} N \Lambda M_{\Lambda} S M_{S} T M_{T}}$ given by

$$
\begin{aligned}
C_{\alpha \beta}^{n l m_{l} N \Lambda M_{\Lambda} S M_{S} T M_{T}}= & \frac{1}{\sqrt{2}}\left[1-(-1)^{l+S+T}\right]\left\langle\frac{1}{2} \tau_{\alpha} \frac{1}{2} \tau_{\beta} \mid T M_{T}\right\rangle\left\langle\frac{1}{2} \sigma_{\alpha} \frac{1}{2} \sigma_{\beta} \mid S M_{S}\right\rangle \\
& \times \sum_{L M_{L}}\left\langle l_{\alpha} m_{l_{\alpha}} l_{\beta} m_{l_{\beta}} \mid L M_{L}\right\rangle\left\langle n l N \Lambda ; L \mid n_{\alpha} l_{\alpha} n_{\beta} l_{\beta} ; L\right\rangle_{\mathrm{SMB}}\left\langle L M_{L} \mid l m_{l} \Lambda M_{\Lambda}\right\rangle,
\end{aligned}
$$

where we use the Talmi-Moshinsky brackets $\langle\mid\rangle_{\mathrm{SMB}}$ [27] to separate out the relative and c.m. coordinates in the products of single-particle wave functions.

After performing the transformation of Eq. (13) for the TBD matrix of Eq. (11), $P_{2}\left(P_{12}\right)$ can be written as

$$
P_{2}\left(P_{12}\right)=\frac{2}{\pi} \sum_{n l m_{l}} \sum_{\Lambda M_{\Lambda}} P_{2}^{n l m_{l} \Lambda M_{\Lambda}}\left(P_{12}\right),
$$

with

$$
\begin{aligned}
P_{2}^{n l m_{l} \Lambda M_{\Lambda}}\left(P_{12}\right)= & \frac{2}{A(A-1)} \sum_{\alpha<\beta} \sum_{N N^{\prime}} \sum_{S M_{S} T M_{T}}\left(C_{\alpha \beta}^{n l m_{l} N^{\prime} \Lambda M_{\Lambda} S M_{S} T M_{T}}\right)^{\dagger} C_{\alpha \beta}^{n l m_{l} N \Lambda M_{\Lambda} S M_{S} T M_{T}} \\
& \times \int d R_{12}^{\prime} R_{12}^{\prime 2} \int d R_{12} R_{12}^{2} j_{\Lambda}\left(P_{12} R_{12}^{\prime}\right) j_{\Lambda}\left(P_{12} R_{12}\right) R_{N^{\prime} \Lambda}\left(\sqrt{2} R_{12}^{\prime}\right) R_{N \Lambda}\left(\sqrt{2} R_{12}\right)
\end{aligned}
$$

A Woods-Saxon basis, for example, first needs to be expanded in a $\mathrm{HO}$ basis before a projection of the type (16) can be made. Using Eqs. (15) and (16), the conditional pair c.m. momentum distribution for a given relative radial quantum number $n$ and relative orbital momentum $l$, can be defined as

$$
P_{2}\left(P_{12} \mid n l=v \lambda\right)=\frac{2}{\pi} \sum_{m_{l}} \sum_{\Lambda M_{\Lambda}} P_{2}^{\nu \lambda m_{l} \Lambda M_{\Lambda}}\left(P_{12}\right) .
$$

Obviously, one has

$$
P_{2}\left(P_{12}\right)=\sum_{\nu \lambda} P_{2}\left(P_{12} \mid n l=v \lambda\right)=\sum_{\lambda} P_{2}\left(P_{12} \mid l=\lambda\right),
$$

where $P_{2}\left(P_{12} \mid l=\lambda\right)$ is the conditional pair c.m. momentum distribution for $l=\lambda$.

A symmetric correlation operator $\widehat{\mathcal{G}}$ can be applied to the IPM wave function of Eq. (10) in order to obtain a realistic ground-state wave function [15,28-30]

$$
\left|\Psi_{A}\right\rangle=\frac{1}{\sqrt{\left\langle\Psi_{A}^{\mathrm{IPM}}\left|\widehat{\mathcal{G}}^{\dagger} \widehat{\mathcal{G}}\right| \Psi_{A}^{\mathrm{IPM}}\right\rangle}} \widehat{\mathcal{G}}\left|\Psi_{A}^{\mathrm{IPM}}\right\rangle .
$$

The operator $\widehat{\mathcal{G}}$ is complicated but as far as the SRC are concerned, it is dominated by the central, tensor, and spinisospin correlations $[31,32]$

$$
\widehat{\mathcal{G}} \approx \widehat{\mathcal{S}}\left[\prod_{i<j=1}^{A}\left(1+\hat{\imath}\left(\vec{x}_{i}, \vec{x}_{j}\right)\right)\right],
$$

with $\widehat{\mathcal{S}}$ the symmetrization operator and

$$
\begin{aligned}
\hat{\imath}\left(\vec{x}_{1}, \vec{x}_{2}\right)= & -g_{c}\left(r_{12}\right)+f_{t \tau}\left(r_{12}\right) S_{12} \vec{\tau}_{1} \cdot \vec{\tau}_{2} \\
& +f_{\sigma \tau}\left(r_{12}\right) \vec{\sigma}_{1} \cdot \vec{\sigma}_{2} \vec{\tau}_{1} \cdot \vec{\tau}_{2},
\end{aligned}
$$

where $g_{c}\left(r_{12}\right), f_{t \tau}\left(r_{12}\right), f_{\sigma \tau}\left(r_{12}\right)$ are the central, tensor, and spin-isospin correlation functions, and $S_{12}$ the tensor operator. The sign convention of $-g_{c}\left(r_{12}\right)$ in Eq. (21) implies that $\lim _{r_{12} \rightarrow 0} g_{c}\left(r_{12}\right)=g_{0}\left(0<g_{0} \leqslant 1\right)$. We stress that the correlation functions cannot be considered as universal [29]. They depend for example on the choices made with regard to the nucleonnucleon interaction, the single-particle basis, and the manybody approximation scheme.

With Eq. (19), the intrinsic complexity stemming from the nuclear correlations is shifted from the wave functions to the transition operators. For example, the ground-state matrix element with a two-body operator $\hat{\mathcal{O}}^{[2]}$ adopts the form

$$
\begin{aligned}
\left\langle\Psi_{A}\left|\hat{\mathcal{O}}^{[2]}\right| \Psi_{A}\right\rangle= & \frac{1}{\left\langle\Psi_{A}^{\mathrm{IPM}}\left|\widehat{\mathcal{G}}^{\dagger} \widehat{\mathcal{G}}\right| \Psi_{A}^{\mathrm{IPM}}\right\rangle} \\
& \times\left\langle\Psi_{A}^{\mathrm{IPM}}\left|\widehat{\mathcal{G}}^{\dagger} \hat{\mathcal{O}}^{[2]} \widehat{\mathcal{G}}\right| \Psi_{A}^{\mathrm{IPM}}\right\rangle,
\end{aligned}
$$

whereby high-order many-body operators are generated. Throughout this work we adopt the two-body cluster (TBC) approximation, which amounts to discarding all terms in $\widehat{\mathcal{G}}^{\dagger} \hat{\mathcal{O}}^{[2]} \widehat{\mathcal{G}}$ except those in which the transition operator and the correlators act on the same pair of particles. In this lowest-order cluster expansion the matrix element of Eq. (22) becomes with the aid of Eq. (20)

$$
\begin{aligned}
\left\langle\Psi_{A}\left|\hat{\mathcal{O}}^{[2]}\right| \Psi_{A}\right\rangle & \approx \frac{1}{\left\langle\Psi_{A} \mid \Psi_{A}\right\rangle}\left\langle\Psi_{A}^{\mathrm{IPM}}\right| \sum_{i<j=1}^{A}\left(1+\hat{\imath}\left(\vec{x}_{i}, \vec{x}_{j}\right)\right)^{\dagger} \hat{\mathcal{O}}^{[2]}(i, j) \\
& \times\left(1+\hat{\imath}\left(\vec{x}_{i}, \vec{x}_{j}\right)\right)\left|\Psi_{A}^{\mathrm{IPM}}\right\rangle \\
= & \frac{1}{\left\langle\Psi_{A} \mid \Psi_{A}\right\rangle} \times\left[\left\langle\Psi_{A}^{\mathrm{IPM}}\left|\hat{\mathcal{O}}^{[2]}\right| \Psi_{A}^{\mathrm{IPM}}\right\rangle+\mathrm{TBC} \text { corrections }\right] .
\end{aligned}
$$

In this expansion, the matrix element is written as the sum of the bare (or IPM) contribution and the TBC corrections to it. The $P_{2}\left(P_{12}\right)$ and $n_{2}\left(k_{12}\right)$ of Eqs. (8) and (9) can be computed with the aid of the Eq. (23) using the transition operators 


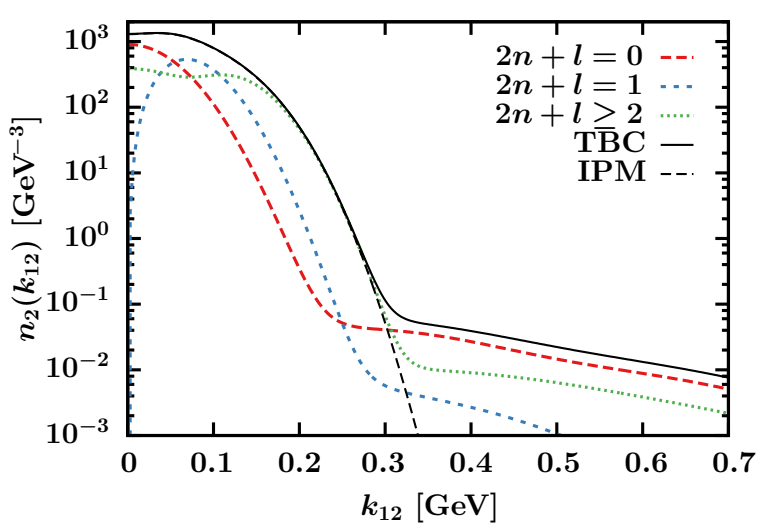

FIG. 1. (Color online) The momentum dependence of the computed $n_{2}^{2 n+l}\left(k_{12}\right), n_{2}^{\mathrm{TBC}}\left(k_{12}\right)$, and $n_{2}^{\mathrm{IPM}}\left(k_{12}\right)$ for ${ }^{56} \mathrm{Fe}$ in a $\mathrm{HO}$ basis. In order to quantify the effect of SRC we have used the $g_{c}\left(r_{12}\right)$ of Ref. [33] and the $f_{t \tau}\left(r_{12}\right), f_{\sigma \tau}\left(r_{12}\right)$ of Ref. [28].

$\delta\left(\vec{P}_{i j}-\left(\vec{k}_{i}+\vec{k}_{j}\right)\right)$ and $\delta\left(\vec{k}_{i j}-\frac{\vec{k}_{i}-\vec{k}_{j}}{2}\right)$. As the $\hat{\imath}$ involves only relative coordinates, the $P_{2}\left(P_{12}\right)$ is not affected by the SRC corrections in the TBC approximation. We define $n_{2}^{\mathrm{IPM}}\left(k_{12}\right)$ as the IPM contribution of $n_{2}\left(k_{12}\right)$ and $n_{2}^{\mathrm{TBC}}\left(k_{12}\right)$ the result obtained with Eq. (23). Accordingly, $n_{2}^{\mathrm{TBC}}\left(k_{12}\right)=n_{2}^{\mathrm{IPM}}\left(k_{12}\right)+$ TBC corrections. For $n_{2}^{\mathrm{TBC}}\left(k_{12}\right)$ the denominator $\left\langle\Psi_{A} \mid \Psi_{A}\right\rangle$ in Eq. (23) can be numerically computed by imposing the normalization conditions: $\int d k_{12} n_{2}^{\mathrm{TBC}}\left(k_{12}\right) k_{12}^{2}=1$. As in Eqs. (7) and (17), one can introduce projection operators, and select the contributions to $n_{2}^{\mathrm{TBC}}\left(k_{12}\right)$ stemming from particular quantum numbers $(n l)$ of the relative two-nucleon wave functions in $\Psi_{A}^{\mathrm{IPM}}$. We define $n_{2}^{2 n+l}\left(k_{12}\right)$ as the contribution to $n_{2}^{\mathrm{TBC}}$ considering only $(n l)$ configurations in $\Psi_{A}^{\mathrm{IPM}}$ with constant $2 n+l$. Obviously, one has

$$
\sum_{2 n+l} n_{2}^{2 n+l}\left(k_{12}\right)=n_{2}^{\mathrm{TBC}}\left(k_{12}\right) \text {. }
$$

The computed $n_{2}^{2 n+l}, n_{2}^{\mathrm{TBC}}$ and $n_{2}^{\mathrm{IPM}}$ for ${ }^{56} \mathrm{Fe}$ are shown in Fig. 1. Below the Fermi momentum $k_{F}$, the effect of the correlation operator is negligible and $n_{2}^{\mathrm{IPM}}\left(k_{12}\right) \approx n_{2}^{\mathrm{TBC}}\left(k_{12}\right)$. For $k_{12}>k_{F}, n_{2}^{\mathrm{IPM}}\left(k_{12}\right)$ drops rapidly while $n_{2}^{\mathrm{TBC}}\left(k_{12}\right)$ exhibits the SRC related high momentum tail. The tail is dominated by the $2 n+l=0$ configurations. This indicates that most of the SRC are dynamically generated through the operation of the correlation operators on $n l=00$ IPM pairs.

In Sec. III, it is shown that in the limit of vanishing FSIs the factorization function of the exclusive $A\left(e, e^{\prime} p N\right)$ cross section is $P_{2}\left(P_{12} \mid n l=00\right)$. In Figs. 2 and 3, we display the computed $P_{2}\left(P_{12}\right)$ and $P_{2}\left(P_{12} \mid n l=00\right)$ for the $p p$ and $p n$ pairs in ${ }^{12} \mathrm{C},{ }^{27} \mathrm{Al},{ }^{56} \mathrm{Fe}$, and ${ }^{208} \mathrm{~Pb}$. The relative weight of the $(n l=00)$ in the total c.m. distribution decreases spectacularly with increasing mass number $A$. This will reflect itself in the mass dependence of the $A\left(e, e^{\prime} N N\right)$ cross sections which are predicted to scale much softer than $A^{2}$. The $(n l=00)$ pairs are strongly localized in space which enlarges the $P_{2}\left(P_{12} \mid n l=00\right)$ width relative to the $P_{2}\left(P_{12}\right)$ one. The mass dependence of the normalized $P_{2}\left(P_{12}\right)$ reflects itself in a modest growth of the width of the distribution. For the light nuclei ${ }^{12} \mathrm{C}$ and ${ }^{27} \mathrm{Al}$, the $p p$ and $p n$ c.m. distributions look very similar.

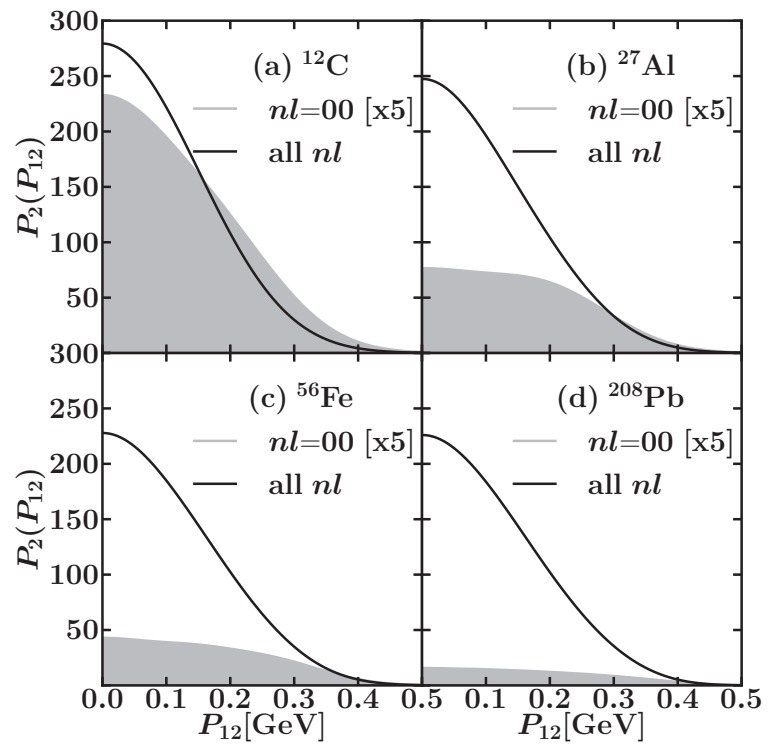

FIG. 2. The momentum dependence of $P_{2}\left(P_{12}\right)$ and the $P_{2}\left(P_{12} \mid n l=00\right)$ for $p p$ pairs in different nuclei. The adopted normalization convention is that $\int_{0}^{\infty} d P_{12} P_{12}^{2} P_{2}\left(P_{12}\right)=1$. Note that only the $p p$ contributions to $P_{2}\left(P_{12}\right)$ are considered when performing the integral. The results are obtained in a $\mathrm{HO}$ basis.

At first sight the computed $P_{2}\left(P_{12}\right)$ for the $p p$ and $p n$ pairs in Figs. 2 and 3 look very Gaussian. In what follows, we use the moments to quantify the non-Gaussianity of the $P_{2}$. The first moment, or mean, of a distribution $F(x)$ is defined as

$$
\mu_{1}=\mu=\frac{\int_{D} x F(x) d x}{\int_{D} F(x) d x}
$$

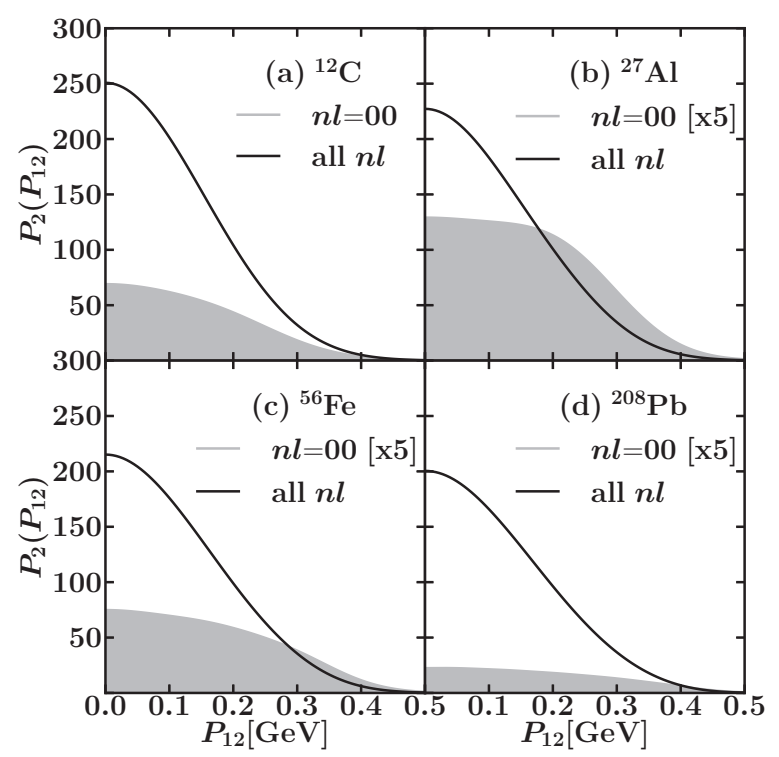

FIG. 3. As in Fig. 2 but for pn pairs. 
TABLE I. The moments of the $P_{2, x}\left(P_{12, x}\right)$ and the $P_{2, x}\left(P_{12, x} \mid n l=00\right)$ distributions for $p p$ pairs as computed in a HO and WS single-particle basis for various nuclei.

\begin{tabular}{|c|c|c|c|c|c|c|c|}
\hline & & \multicolumn{3}{|c|}{$\mathrm{HO}$} & \multicolumn{3}{|c|}{ WS } \\
\hline${ }^{12} \mathrm{C}$ & $P_{2, x}\left(P_{12, x} \mid n l=00\right)$ & 156 & 0.00 & -0.25 & 158 & 0.00 & -0.28 \\
\hline${ }^{12} \mathrm{C}$ & $P_{2, x}\left(P_{12, x}\right)$ & 140 & -0.01 & -0.12 & 142 & -0.01 & -0.05 \\
\hline${ }^{27} \mathrm{Al}$ & $P_{2, x}\left(P_{12, x} \mid n l=00\right)$ & 164 & 0.00 & -0.45 & 168 & 0.00 & -0.45 \\
\hline${ }^{27} \mathrm{Al}$ & $P_{2, x}\left(P_{12, x}\right)$ & 144 & -0.01 & -0.20 & 148 & -0.01 & -0.20 \\
\hline${ }^{208} \mathrm{~Pb}$ & $P_{2, x}\left(P_{12, x} \mid n l=00\right)$ & 178 & 0.00 & -0.58 & 177 & 0.00 & -0.63 \\
\hline${ }^{208} \mathrm{~Pb}$ & $P_{2, x}\left(P_{12, x}\right)$ & 145 & 0.00 & -0.31 & 146 & 0.00 & -0.31 \\
\hline
\end{tabular}

where $D$ is the domain of the distribution. For $m>1$, we define the central moments as

$$
\mu_{m}=\frac{\int_{D}(x-\mu)^{m} F(x) d x}{\int_{D} F(x) d x} .
$$

The width is defined as $\sigma=\sqrt{\mu_{2}}$. With regard to $\mu_{3}$ and $\mu_{4}$, it is common practice to describe a distribution with the skewness $\gamma_{1}$ and excess kurtosis $\kappa$

$$
\begin{aligned}
\gamma_{1} & \equiv \frac{\mu_{3}}{\sigma^{3}}, \\
\kappa & \equiv \frac{\mu_{4}}{\sigma^{4}}-3,
\end{aligned}
$$

which are both vanishing for a Gaussian distribution.

For a spherically symmetric distribution, one can derive the distributions $P_{2, i}\left(P_{12, i}\right)(i=x, y, z)$ along the axes from $P_{12}^{2} P_{2}\left(P_{12}=\sqrt{P_{12, x}^{2}+P_{12, y}^{2}+P_{12, z}^{2}}\right)$. Gaussian $P_{2, i}$ give rise to a $P_{12}^{2} P_{2}\left(P_{12, i}\right)$ of the Maxwell-Boltzmann type.

Table I shows the computed moments of the $P_{2, x}\left(P_{12, x} \mid n l=\right.$ $00)$ and $P_{2, x}\left(P_{12, x}\right)$ distributions for $p p$ pairs. These results are obtained with HO and Woods-Saxon (WS) single-particle wave functions. We find that the c.m. distributions are not perfectly Gaussian and that the non-Gaussianity grows with $A$. The values of the widths are only moderately sensitive to the single-particle basis used. The WS widths are larger by a few percent than the $\mathrm{HO}$ ones.

In Fig. 4, the calculated widths of the $P_{2, x}\left(P_{12, x}\right)$ and $P_{2, x}\left(P_{12, x} \mid l\right)$ are shown for $p p, n n$, and $n p$ pairs. For the $n p$ pairs we discriminate between singlet $(S=0)$ and triplet $(S=$ 1) spin states. From Fig. 4 we draw the following conclusions. The width of the $P_{2, x}\left(P_{12} \mid l\right)$ depends on $l$. For $l=0$ and $n p$ pairs, the width of $P_{2, x}(P \mid l)$ is almost independent of $S$. For heavy nuclei there is a substantial difference in the width of the $P_{2, x}(P \mid l=0)$ for $p p, n n$, and $n p$ pairs but for light nuclei this is not the case. A similar but smaller dependence on the width is found for $n$ at fixed $l$, the width of $P_{2}\left(P_{12} \mid n l\right)$ decreases for increasing $n$. We conclude that from the width of the c.m. distribution of the pairs one can infer information about their relative orbital momentum.

\section{FACTORIZATION OF THE TWO-NUCLEON KNOCKOUT CROSS SECTION}

It is well known that the fivefold differential cross section for the exclusive $A\left(e, e^{\prime} p\right) A-1$ reaction under quasifree kinematics with $A-1$ spectators

$$
\begin{gathered}
\gamma^{*}(q)+A-1\left(p_{A-1}\right)+N\left(k_{1}\right) \\
\longrightarrow A-1\left(p_{A-1}\right)+N\left(p_{1}\right),
\end{gathered}
$$

factorizes as

$$
d^{5} \sigma\left(e, e^{\prime} p\right)=K_{e p} \sigma_{e p} P_{1}\left(\vec{k}_{m}, E_{m}\right) .
$$

Here, $K_{e p}$ is a kinematical factor and $\sigma_{e p}$ the off-shell electronproton cross section. Further, $\vec{k}_{m}=-\vec{p}_{A-1}=\vec{k}_{1}$ is the missing momentum and $E_{m}=q^{0}-T_{p_{1}}-T_{A-1}$ the missing energy, whereby $T_{A-1}$ and $T_{p_{1}}$ are the kinetic energy of the recoiling nucleus and ejected nucleon. The $P_{1}(\vec{k}, E)$ is the one-body spectral function and is associated with the combined probability of removing a proton with momentum $\vec{k}$ from the ground

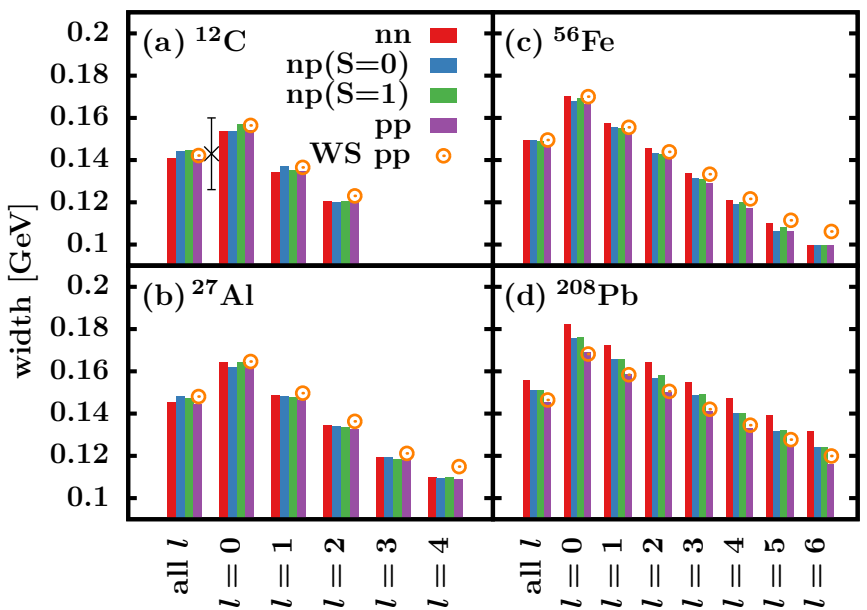

FIG. 4. (Color online) Computed widths of the $P_{2, x}\left(P_{12, x}\right)$ (denoted as "all $l$ ") and $P_{2, x}\left(P_{12, x} \mid l\right)$ distributions for $p p, n n, n p(S=0)$, and $n p(S=1)$ pairs in ${ }^{12} \mathrm{C},{ }^{27} \mathrm{Al},{ }^{56} \mathrm{Fe},{ }^{208} \mathrm{~Pb}$. Unless stated otherwise the results are obtained in a $\mathrm{HO}$ basis. For $p p$ pairs we also display results for a WS basis (denoted as "WS pp"). The black cross is the experimental result from Ref. [1]. 
state of $A$ and of finding the residual $A-1$ nucleus at excitation energy $E$ (measured relative to the ground state of the target nucleus). The factorization is exact in a nonrelativistic reaction model with $A-1$ spectators and vanishing FSIs [34]. The validity of the spectator approximation requires that the $E_{m}$ is confined to low values, corresponding to states with a predominant one-hole character relative to the ground state of the target nucleus $A$.

Below, it is shown that also the $A\left(e, e^{\prime} p N\right)$ differential cross section factorizes under certain assumptions. The factorization function is connected to the c.m. motion of close-proximity pairs. In Ref. [35] the factorization function is introduced as the so-called decay function. In Ref. [36] a factorized expression for the $A\left(e, e^{\prime} p p\right)$ cross section has been derived. Thereby, in computing the matrix elements, all FSI effects have been neglected and the zero-range approximation $\left(\lim _{r_{12} \rightarrow 0}\right)$ has been adopted. A ${ }^{12} \mathrm{C}\left(e, e^{\prime} p p\right)$ experiment conducted at the Mainz Microtron (MAMI) [37] showed very good quantitative agreement with the predicted diproton pair c.m. momentum factorization up to momenta of about $500 \mathrm{MeV}$. Here, the formalism of Ref. [36] is extended to include the effect of FSIs and to soften the zero-range approximation. Note that the limit $\lim _{r_{12} \rightarrow 0}$ effectively amounts to projecting on states with vanishing relative orbital momentum.

We consider exclusive $A\left(e, e^{\prime} N N\right)$ reactions in the spectator approximation with a virtual photon coupling to a correlated pair $N\left(k_{1}\right) N\left(k_{2}\right)$

$$
\begin{aligned}
& \gamma^{*}(q)+A-2\left(p_{A-2}\right)+N\left(k_{1}\right) N\left(k_{2}\right) \\
& \longrightarrow A-2\left(p_{A-2}\right)+N\left(p_{1}\right)+N\left(p_{2}\right) .
\end{aligned}
$$

In a nonrelativistic treatment, the corresponding matrix element is given by

$$
\begin{aligned}
\mathcal{M}^{\mu}= & \int d \vec{x}_{1} \int d \vec{x}_{2}\left[\chi_{s_{1}}^{\dagger}\left(\vec{\sigma}_{1}\right) \xi_{t_{1}}^{\dagger}\left(\vec{\tau}_{1}\right) \chi_{s_{2}}^{\dagger}\left(\vec{\sigma}_{2}\right) \xi_{t_{2}}^{\dagger}\left(\vec{\tau}_{2}\right)\right. \\
& \left.\times e^{-i \vec{p}_{1} \cdot \vec{r}_{1}} e^{-i \vec{p}_{2} \cdot \vec{r}_{2}}-(1 \leftrightarrow 2)\right] \\
& \times \mathcal{F}_{\mathrm{FSI}}^{\dagger}\left(\vec{r}_{1}, \vec{r}_{2}\right) \hat{\mathcal{O}}^{\mu}\left(\vec{x}_{1}, \vec{x}_{2}\right) \phi_{\alpha_{1}}\left(\vec{x}_{1}\right) \phi_{\alpha_{2}}\left(\vec{x}_{2}\right) .
\end{aligned}
$$

Here, $s_{i}\left(t_{i}\right)$ are the spin (isospin) projection of the outgoing nucleons. Further, $\mathcal{F}_{\mathrm{FSI}}\left(\vec{r}_{1}, \vec{r}_{2}\right)$ is an operator encoding the FSIs for a reaction where two nucleons are brought into the continuum at the spatial localizations $\vec{r}_{1}$ and $\vec{r}_{2}$, respectively. We assume that $\mathcal{F}_{\mathrm{FSI}}$ does not depend on the spin and isospin d.o.f, which is a fair approximation at higher energies. The amplitude of Eq. (32) refers to the physical situation whereby, as a result of virtual-photon excitation, two nucleons are excited from bound states $\alpha_{1} \alpha_{2}$ into continuum states.

In Eq. (32), the effect of the correlations is implemented in the TBC approximation by means of a symmetric two-body operator $[29,31]$

$$
\begin{aligned}
& \hat{\mathcal{O}}^{\mu}\left(\vec{x}_{1}, \vec{x}_{2}\right) \\
& \quad=\left[e^{i \vec{q} \cdot \vec{r}_{1}} \Gamma_{\gamma^{\star} N}^{\mu}\left(\vec{x}_{1}\right)+e^{i \vec{q} \cdot \vec{r}_{2}} \Gamma_{\gamma^{\star} N}^{\mu}\left(\vec{x}_{2}\right)\right] \hat{\imath}\left(\vec{x}_{1}, \vec{x}_{2}\right),
\end{aligned}
$$

where the operator $\hat{\imath}\left(\vec{x}_{1}, \vec{x}_{2}\right)$ has been defined in Eq. (21) and $\vec{q}$ is the three-momentum of the virtual photon. The $\Gamma_{\gamma^{\star} N}^{\mu}\left(\vec{x}_{i}\right)$ denotes the one-body virtual photon coupling to a bound nucleon with coordinate $\vec{x}_{i}$ (includes the spatial, spin, and isospin d.o.f.). Equation (33) can be interpreted as the SRC-corrected photonucleon coupling which operates on IPM many-body wave functions.

The amplitude of Eq. (32) involves four contributions schematically shown in Fig. 5. For the sake of brevity, in the following we consider the term of Fig. 5(a) with a photon-nucleon coupling on coordinate $\vec{r}_{1}$ and the outgoing nucleon with momentum $\vec{p}_{1}$ directly attached to this vertex. The corresponding amplitude is denoted by $\mathcal{M}_{a}^{\mu}$. The other three terms in Fig. 5 follow a similar derivation.

In a $\mathrm{HO}$ single-particle basis, one can write

$$
\begin{aligned}
\mathcal{M}_{a}^{\mu}= & \int d \vec{r}_{1} \int d \vec{r}_{2} e^{-i\left(\vec{p}_{1}-\vec{q}\right) \cdot \vec{r}_{1}} e^{-i \vec{p}_{2} \cdot \vec{r}_{2}} \mathcal{F}_{\mathrm{FSI}}^{\dagger}\left(\vec{r}_{1}, \vec{r}_{2}\right) \\
& \times\left\langle s_{1} t_{1}, s_{2} t_{2}\left|\Gamma_{\gamma^{\star} N}^{\mu}\left(\vec{x}_{1}\right) \hat{\imath}\left(\vec{x}_{1}, \vec{x}_{2}\right)\right| \sigma_{1} \tau_{1}, \sigma_{2} \tau_{2}\right\rangle \\
& \times \psi_{n_{1} l_{1} m_{l_{1}}}\left(\vec{r}_{1}\right) \psi_{n_{2} l_{2} m_{l_{2}}}\left(\vec{r}_{2}\right),
\end{aligned}
$$

where $\sigma_{i}\left(\tau_{i}\right)$ are the spin (isospin) quantum numbers of the bound states. Further, $\psi_{n_{1} l_{1} m_{l_{1}}}$ and $\psi_{n_{2} l_{2} m_{l_{2}}}$ are the radial HO wave functions as introduced in Eq. (12).

Similar to the Eq. (13), we apply the Talmi-Moshinsky brackets $\langle\mid\rangle_{\mathrm{SMB}}$ [27] to transform Eq. (34) to relative and c.m. radial coordinates to obtain

$$
\begin{aligned}
\mathcal{M}_{a}^{\mu}= & \sum_{L M_{L}} \sum_{\substack{n l m_{l} \\
N \Lambda M_{\Lambda}}} \int d \vec{r}_{12} \int d \vec{R}_{12} e^{-i \vec{P}_{12} \cdot \vec{R}_{12}} e^{-i \vec{k}^{-} \cdot \vec{r}_{12}} \mathcal{F}_{\mathrm{FSI}}^{\dagger}\left(\vec{R}_{12}+\frac{\vec{r}_{12}}{2}, \vec{R}_{12}-\frac{\vec{r}_{12}}{2}\right) \psi_{n l m_{l}}\left(\frac{\vec{r}_{12}}{\sqrt{2}}\right) \psi_{N \Lambda M_{\Lambda}}\left(\sqrt{2} \vec{R}_{12}\right) \\
& \times\left\langle l_{1} m_{l_{1}} l_{2} m_{l_{2}} \mid L M_{L}\right\rangle\left\langle l m_{l} \Lambda M_{\Lambda} \mid L M_{L}\right\rangle\left\langle n l N \Lambda ; L \mid n_{1} l_{1} n_{2} l_{2} ; L\right\rangle_{\mathrm{SMB}}\left\langle s_{1} t_{1}, s_{2} t_{2}\left|\Gamma_{\gamma^{*} N}^{\mu}\left(\vec{x}_{1}\right) \hat{\imath}\left(\vec{x}_{1}, \vec{x}_{2}\right)\right| \sigma_{1} \tau_{1}, \sigma_{2} \tau_{2}\right\rangle,
\end{aligned}
$$

where $\vec{P}_{12}=\vec{p}_{1}+\vec{p}_{2}-\vec{q}, \vec{k}^{\mp}=\frac{\vec{p}_{1}-\vec{p}_{2}}{2} \mp \frac{\vec{q}}{2}$.

In Eq. (35) the sum over the relative quantum numbers is dominated by $(n l=00)$. This is based on the observation that typical correlation operators act over relatively short internucleon distances and mostly affect the $(n l=00)$ components of the $\psi_{n l m_{l}}$ wave functions. For a more detailed explanation we refer to the discussion of Fig. 1 in Sec. II and Refs. [20,21].
For close-proximity nucleons one can set $\vec{r}_{12} \approx \overrightarrow{0}$ in the FSI operator:

$$
\begin{aligned}
\mathcal{F}_{\mathrm{FSI}}\left(\vec{r}_{1}, \vec{r}_{2}\right) & =\mathcal{F}_{\mathrm{FSI}}\left(\vec{R}_{12}+\frac{\vec{r}_{12}}{2}, \vec{R}_{12}-\frac{\vec{r}_{12}}{2}\right) \\
& \approx \mathcal{F}_{\mathrm{FSI}}\left(\vec{R}_{12}, \vec{R}_{12}\right) .
\end{aligned}
$$



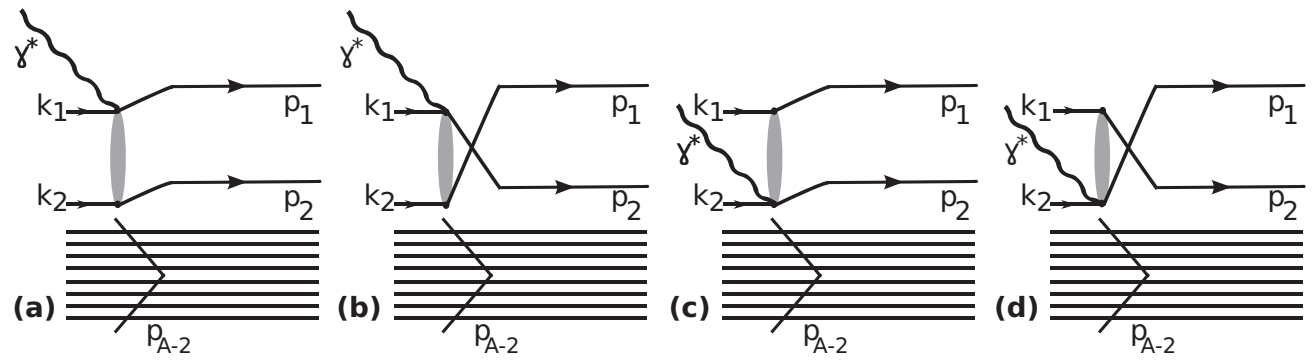

FIG. 5. The four contributions to the $A\left(e, e^{\prime} N N\right)$ amplitude of Eq. (32).

This approximation amounts to computing the effect of FSIs as if the the two nucleons are brought into the continuum at the same spatial point (determined by the c.m. coordinate of the pair), which is very reasonable for close-proximity nucleons. With the above assumptions one arrives at the expression for the matrix element

$$
\begin{aligned}
\mathcal{M}_{a}^{\mu} \approx & \left\langle s_{1} t_{1}, s_{2} t_{2}\left|\widehat{\Gamma}_{\gamma^{*} N}^{\mu}\left(\vec{k}^{-}\right)\right| \sigma_{1} \tau_{1}, \sigma_{2} \tau_{2}\right\rangle \\
& \times \sum_{N \Lambda M_{\Lambda}}\left\langle l_{1} m_{l_{1}} l_{2} m_{l_{2}} \mid \Lambda M_{\Lambda}\right\rangle\left\langle 00 N \Lambda ; \Lambda \mid n_{1} l_{1} n_{2} l_{2} ; \Lambda\right\rangle_{\mathrm{SMB}} \\
& \times \int d \vec{R}_{12} e^{-i \vec{P}_{12} \cdot \vec{R}_{12}} \mathcal{F}_{\mathrm{FSI}}^{\dagger}\left(\vec{R}_{12}, \vec{R}_{12}\right) \psi_{N \Lambda M_{\Lambda}}\left(\sqrt{2} \vec{R}_{12}\right),
\end{aligned}
$$

with

$$
\widehat{\Gamma}_{\gamma^{\star} N}^{\mu}(\vec{p}) \equiv \int d \vec{r}_{12} e^{-i \vec{p} \cdot \vec{r}_{12}} \psi_{000}\left(\frac{\vec{r}_{12}}{\sqrt{2}}\right) \Gamma_{\gamma^{\star} N}^{\mu}\left(\vec{x}_{1}\right) \hat{\imath}\left(\vec{x}_{1}, \vec{x}_{2}\right)
$$

In deriving the Eq. (37), we have separated the integration over the spatial and spin-isospin d.o.f. In addition, use has been made of the fact that the operator $\hat{\imath}\left(\vec{x}_{1}, \vec{x}_{2}\right)$ of Eq. (21) does not depend on the c.m. coordinate $\vec{R}_{12}$. The most striking feature of Eq. (37) is the factorization of the amplitude in a term connected to the c.m. motion of the initial pair and a term which contains the full complexity of the photon-nucleon coupling to a correlated pair.

After summing the four terms that contribute to Eq. (32) and squaring the matrix element, the eightfold differential cross section factorizes according to

$$
d^{8} \sigma\left(e, e^{\prime} N N\right)=K_{e N N} \sigma_{e 2 N} F_{n_{1} l_{1}, n_{2} l_{2}}^{D}\left(\vec{P}_{12}\right),
$$

with $K_{e N N}$ a kinematic factor. Further, the off-shell electrontwo-nucleon cross section is given by

$$
\sigma_{e 2 N} \propto L_{\mu \nu} \sum_{\substack{s_{1} s_{2} \sigma_{1} \sigma_{2} \\ \tau_{1} \tau_{2}}} J^{\mu}\left(J^{\nu}\right)^{\dagger},
$$

with $L_{\mu \nu}$ the leptonic tensor and $J^{\mu}$ the hadronic current given by

$$
\begin{aligned}
J^{\mu}= & \left\langle s_{1} t_{1}, s_{2} t_{2}\left|\widehat{\Gamma}_{\gamma^{\star} N}^{\mu}\left(\vec{k}^{-}\right)\right| \sigma_{1} \tau_{1}, \sigma_{2} \tau_{2}\right\rangle \\
& -\left\langle s_{2} t_{2}, s_{1} t_{1}\left|\widehat{\Gamma}_{\gamma^{\star} N}^{\mu}\left(\vec{k}^{+}\right)\right| \sigma_{1} \tau_{1}, \sigma_{2} \tau_{2}\right\rangle \\
& +\left\langle s_{1} t_{1}, s_{2} t_{2}\left|\widehat{\Gamma}_{\gamma^{\star} N}^{\mu}\left(\vec{k}^{+}\right)\right| \sigma_{1} \tau_{1}, \sigma_{2} \tau_{2}\right\rangle \\
& -\left\langle s_{2} t_{2}, s_{1} t_{1}\left|\widehat{\Gamma}_{\gamma^{\star} N}^{\mu}\left(\vec{k}^{-}\right)\right| \sigma_{1} \tau_{1}, \sigma_{2} \tau_{2}\right\rangle .
\end{aligned}
$$

The factorization function $F_{n_{1} l_{1}, n_{2} l_{2}}^{D}\left(\vec{P}_{12}\right)$ in Eq. (39) can be associated with the distorted c.m. momentum distribution of pairs in a relative $(n l=00)$ state of the nucleus $A$

$$
\begin{aligned}
& F_{n_{1} l_{1}, n_{2} l_{2}}^{D}\left(\vec{P}_{12}\right) \\
& =4 \sum_{m_{l_{1}} m_{l_{2}}} \mid \sum_{N \Lambda M_{\Lambda}} \int d \vec{R}_{12} e^{-i \vec{P}_{12} \cdot \vec{R}_{12}} \\
& \quad \times\left\langle l_{1} m_{l_{1}} l_{2} m_{l_{2}} \mid \Lambda M_{\Lambda}\right\rangle\left\langle n_{1} l_{1} n_{2} l_{2} ; \Lambda \mid 00 N \Lambda ; \Lambda\right\rangle_{\mathrm{SMB}} \\
& \quad \times\left.\mathcal{F}_{\mathrm{FSI}}^{\dagger}\left(\vec{R}_{12}, \vec{R}_{12}\right) \psi_{N \Lambda M_{\Lambda}}\left(\sqrt{2} \vec{R}_{12}\right)\right|^{2},
\end{aligned}
$$

where the factor 4 accounts for the spin degeneracy of the $\mathrm{HO}$ states.

In the limit of vanishing FSIs $\left(\mathcal{F}_{\mathrm{FSI}} \equiv 1\right)$, one has

$$
\begin{aligned}
P_{2}\left(P_{12} \mid n l=00\right)= & \frac{1}{A(A-1)} \frac{3}{(2 \pi)^{3}} \\
& \times \sum_{n_{\alpha} l_{\alpha} n_{\beta} l_{\beta}} \int d \Omega_{P_{12}} F_{n_{\alpha} l_{\alpha}, n_{\beta} l_{\beta}}^{D}\left(\vec{P}_{12}\right) .
\end{aligned}
$$

This establishes a connection between the $A\left(e, e^{\prime} N N\right)$ factorization function and the contribution of pairs with quantum numbers $\left(n_{1} l_{1} n_{2} l_{2}\right)$ to $P_{2}\left(P_{12} \mid n l=00\right)$, illustrated for $p p$ pairs in ${ }^{12} \mathrm{C}$ in Fig. 6.

In the naive IPM, each two-hole (2h) state $\left(n_{1} l_{1}\right)^{-1}\left(n_{2} l_{2}\right)^{-1}$ can be associated with a sharp excitation energy in the

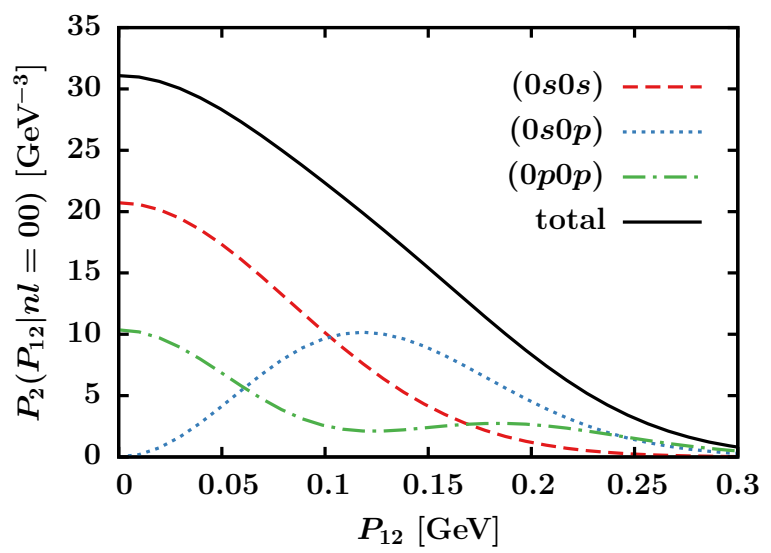

FIG. 6. (Color online) The contribution of the different shellmodel pair combinations to the $P_{2}\left(P_{12} \mid n l=00\right)$ for $p p$ pairs in ${ }^{12} \mathrm{C}$. 
$A-2$ system. In reality, the $2 \mathrm{~h}$ strength corresponding with $\left(n_{1} l_{1}\right)^{-1}\left(n_{2} l_{2}\right)^{-1}$ extends over a wide energy range [38]. Current $A\left(e, e^{\prime} p N\right)$ measurements are performed at $Q^{2}$ values of the order of $\mathrm{GeV}^{2}$ not allowing one to measure cross sections for real exclusive processes as could be done at lower $Q^{2}$ values $[26,39,40]$. Accordingly, rather than probing the individual $2 \mathrm{~h}$ contributions to $P_{2}$, the measured semi-inclusive $A\left(e, e^{\prime} p N\right)$ cross sections can be linked to the $P_{2}\left(P_{12} \mid n l=\right.$ $00)$ which involves a summation over the $2 \mathrm{~h}$ states. From Fig. 6 it can be appreciated that in high-resolution $A\left(e, e^{\prime} p N\right)$ measurements the c.m. distribution depends on the two-hole structure of the discrete final $A-2$ state $[38,39]$.

The $A\left(e, e^{\prime} p\right)$ reaction allows one to access the $P_{1}\left(\vec{k}_{m}, E_{m}\right)$ modulo corrections from FSIs. It is worth stressing that there is no simple analogy for the $A\left(e, e^{\prime} p N\right)$ reaction and that a direct connection with the two-body spectral function $P_{2}\left(\vec{P}_{12}, \vec{k}_{12}, E_{2 m}\right)$ is by no means evident, if not impossible.

\section{MONTE CARLO SIMULATIONS}

In this section, we investigate the implications of the proposed factorization of Eq. (39) for the $A\left(e, e^{\prime} p p\right)$ openingangle and c.m. distributions accessible in typical measurements. We present Monte Carlo simulations for $A\left(e, e^{\prime} p p\right)$ building on the expression (39) suggesting that the magnitude of the cross section is proportional to $P_{2}\left(P_{12} \mid n l=00\right)$. In this section the effects of FSIs are neglected. Its impact will be the subject of Sec. V.

The data-mining effort at CLAS in JLab [41,42] is analyzing exclusive $\left(e, e^{\prime} p N\right)$ for ${ }^{12} \mathrm{C},{ }^{27} \mathrm{Al},{ }^{56} \mathrm{Fe}$, and ${ }^{208} \mathrm{~Pb}$ for a $5.014 \mathrm{GeV}$ unpolarized electron beam [41]. In order to guarantee the exclusive character of the events, cuts are applied to the leading proton: $0.62<\frac{\left|\vec{p}_{1}\right|}{|\vec{q}|}<0.96, \theta_{\vec{p}_{1}, \vec{q}}<25^{\circ}$, and $k_{1}>$ $300 \mathrm{MeV}$. To increase the sensitivity to SRC-driven processes one imposes the kinematic constraints $x_{B}=\frac{Q^{2}}{2 M_{N} \omega}>1.2$ and $Q^{2}>1.4 \mathrm{GeV}^{2}$. We have performed ( $\left.e, e^{\prime} p p\right)$ simulations for all four target nuclei. The electron kinematics are drawn from the measured $x_{B}-Q^{2}$ distributions. We then generate two protons from the phase space by adopting a reaction picture of the type (31) whereby we assume that one nucleon absorbs the virtual photon. This results in a fast leading proton $p_{1}\left(E_{1}, \vec{p}_{1}=\right.$ $\left.\vec{k}_{1}+\vec{q}\right)$ and a recoil proton $p_{2}\left(E_{2}, \vec{p}_{2}=\vec{k}_{2}\right)$, where $\vec{k}_{1}$ and $\vec{k}_{2}$ are the initial proton momenta. The initial c.m. momentum $\vec{P}_{12}=\vec{k}_{1}+\vec{k}_{2}$ is drawn from the computed HO pp pair c.m. momentum distribution $P_{2}\left(P_{12} \mid n l=00\right)$ of Table I. We choose $\vec{k}_{1}$ along the $z$ axis and $\vec{q}$ in the $x z$ plane. The recoil $A-2$ nucleus can have excitation energies between 0 and $80 \mathrm{MeV}$. All $A\left(e, e^{\prime} p p\right)$ results of this section are obtained for $10^{5}$ events which comply with the kinematic cuts.

First, we investigate in how far the factorization function can be addressed after applying kinematic cuts. This can be done by comparing the input and extracted $p p$ c.m. distributions. Figure 7 shows the extracted c.m. distribution from the simulated ${ }^{12} \mathrm{C}\left(e, e^{\prime} p p\right)$ events. The kinematic cuts have a narrowing effect (less than 10\%) on the distributions along the $x$ and $y$ axis. In addition, one observes a shift of roughly $100 \mathrm{MeV}$ and an increase in the non-Gaussianity of

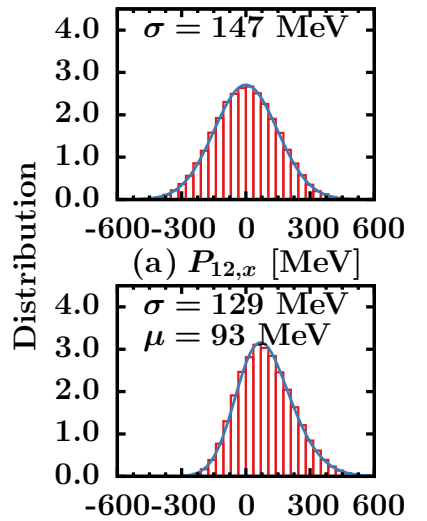

(c) $P_{12, z}[\mathrm{MeV}]$

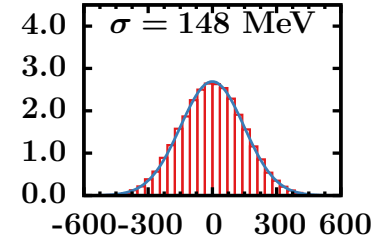

(b) $\boldsymbol{P}_{12, y}[\mathrm{MeV}]$

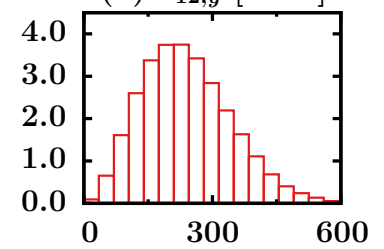

(d) $\left|P_{12}\right|[\mathrm{MeV}]$
FIG. 7. (Color online) Total (bottom right) and directional $p p$ c.m. distributions extracted from the ${ }^{12} \mathrm{C}\left(e, e^{\prime} p p\right)$ simulations in the CLAS kinematics described in the text. The blue solid line is a fit with a skew normal distribution.

the c.m. distribution along the $z$ axis. Similar observations have been made for the other three target nuclei.

We now address the issue whether the extracted c.m. distributions can provide information about the relative quantum numbers of the pairs. To this end, we have performed simulations starting from the assumption that the $\left(e, e^{\prime} p p\right)$ cross section factorizes with $P_{2}\left(P_{12} \mid n l\right)$ for various $n l$ combinations. The results of the simulations are summarized in Table II. The narrowing effect attributed to the kinematic cuts is less significant for $l>0$ pairs. Photon absorption on $l=0$ and $l=1$ pairs leads to differences in the extracted widths of the c.m. momentum distributions of the order of $20 \mathrm{MeV}$, which leads us to conclude that high-accuracy $A\left(e, e^{\prime} p p\right)$ experiments could indeed provide information about the relative orbital angular momentum of the correlated pairs.

Figure 8 shows the simulated opening-angle $(\gamma)$ distributions of the initial-state protons for all four target nuclei considered. The $A\left(e, e^{\prime} p p\right)$ simulations starting from the computed $P_{2}\left(P_{12} \mid n l=00\right)$ and $P_{2}\left(P_{12}\right)$ provide very similar backwardly peaked $\cos \gamma$ distributions. The peak is not due to the kinematic cuts as a uniform c.m. momentum distributions gives rise to a flat $\cos \gamma$ distribution. The shape of the simulated $\cos \gamma$ distributions is hardly target-mass dependent. The peak at 180 degrees in the $\cos \gamma$ distributions conforms with the picture of correlated nucleons moving back to back with high relative and low c.m. momentum.

TABLE II. The width of the c.m. distribution along the $x$ axis for $p p$ pairs with different relative orbital momentum $l . \sigma_{x}^{i}$ is the width used as input parameter in the ${ }^{12} \mathrm{C}\left(e, e^{\prime} p p\right)$ simulations. The $\sigma_{x}^{f}$ is the width extracted after the simulation.

\begin{tabular}{lccccc}
\hline \hline & $n l=00$ & $l=0$ & $l=1$ & $l=2$ & all $l$ \\
\hline$\sigma_{x}^{i}(\mathrm{MeV})$ & 156 & 154 & 135 & 121 & 140 \\
$\sigma_{x}^{f}(\mathrm{MeV})$ & 147 & 145 & 130 & 118 & 134 \\
\hline \hline
\end{tabular}




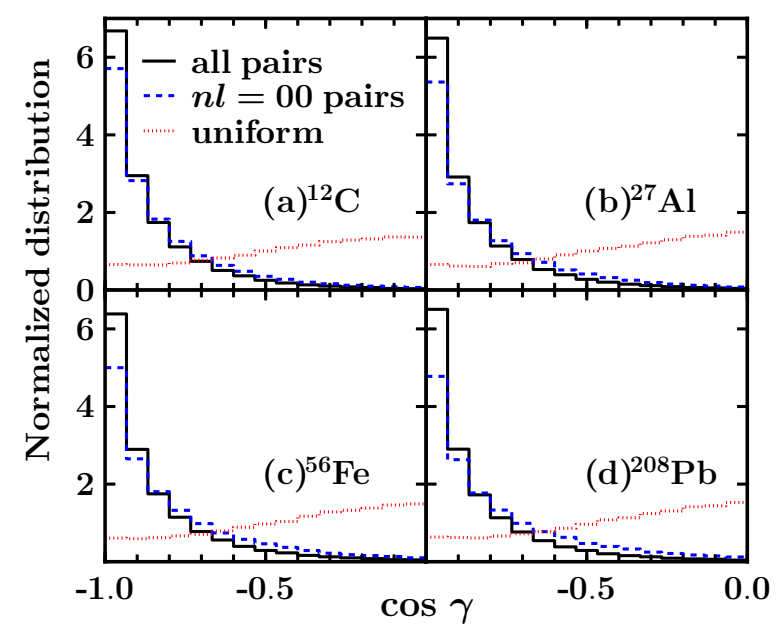

FIG. 8. (Color online) The opening angle distribution of the simulated $A\left(e, e^{\prime} p p\right)$ events in the kinematics described in the text. The black solid, blue dashed, and red dotted lines are for a reaction picture with an $\left(e, e^{\prime} p p\right)$ cross section proportional to $P_{2}\left(P_{12}\right)$, to $P_{2}\left(P_{12} \mid n l=00\right)$, and to a uniform pair c.m. distribution.

We now turn our attention to an ${ }^{12} \mathrm{C}\left(e, e^{\prime} p p\right)$ measurement probing a restricted part of phase space. The JLab Hall-A ${ }^{12} \mathrm{C}\left(e, e^{\prime} p p\right)$ experiment of Refs. [3,4], used an incident electron beam of $4.672 \mathrm{GeV}$ and three spectrometers. We consider the kinematic settings with $\omega=0.865 \mathrm{GeV}, Q^{2}=2 \mathrm{GeV}^{2}$, $x_{B}=1.2$, and a median missing momentum $p_{m}=0.55 \mathrm{GeV}$. Figure 9 shows the shapes of the simulated and measured $\cos \gamma$ simulations. The proposed factorization for the $A\left(e, e^{\prime} p p\right)$ cross section accounts for the shape of the measured $\cos \gamma$ distribution. We stress that the computed pair c.m. distributions (Table I) are the sole input to the simulations.

\section{FINAL STATE INTERACTIONS}

In this section the impact of FSIs on the proposed factorization function of Eq. (39) is investigated. In order to keep computing times reasonable we limit ourselves to some particular kinematic cases and introduce an additional approximation. We start from Eq. (42) for the distorted momentum distribution

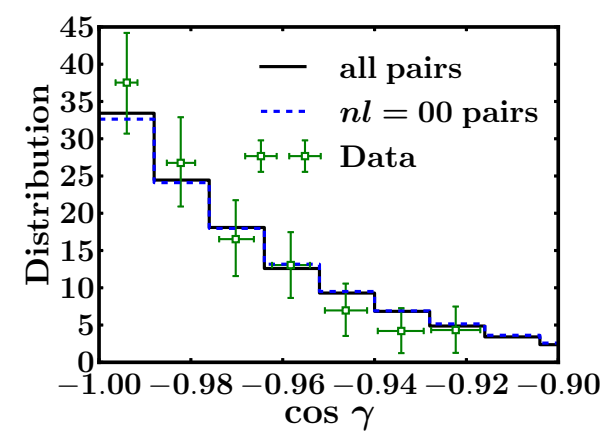

FIG. 9. (Color online) The opening angle distribution of the ${ }^{12} \mathrm{C}\left(e, e^{\prime} p p\right)$ reaction in the kinematics of Ref. [3]. Curve notations of Fig. 8 are used.
$F_{n_{1} l_{1}, n_{2} l_{2}}^{D}\left(\vec{P}_{12}\right)$ and apply the zero-range approximation [36,43] which amounts to setting $\psi_{\alpha_{1}}\left(\vec{r}_{1}\right) \psi_{\alpha_{2}}\left(\vec{r}_{2}\right) \approx \psi_{\alpha_{1}}\left(\vec{R}_{12}\right) \psi_{\alpha_{2}}\left(\vec{R}_{12}\right)$ in Eq. (34). Consequently, we can write

$$
\begin{aligned}
F_{n_{1} l_{1}, n_{2} l_{2}}^{D}\left(\vec{P}_{12}\right)= & 4 \sum_{m_{l_{1} m_{l_{2}}}} \mid \int d \vec{R}_{12} e^{-i \vec{P}_{12} \cdot \vec{R}_{12}} \\
& \times\left.\mathcal{F}_{\mathrm{FSI}}^{\dagger}\left(\vec{R}_{12}, \vec{R}_{12}\right) \psi_{n_{1} l_{1} m_{l_{1}}}\left(\vec{R}_{12}\right) \psi_{n_{2} l_{2} m_{l_{2}}}\left(\vec{R}_{12}\right)\right|^{2} .
\end{aligned}
$$

It is possible to derive a relativized version of this expression [43]

$$
\begin{aligned}
& F_{n_{1} \kappa_{1}, n_{2} \kappa_{2}}^{D}\left(\vec{P}_{12}\right) \\
& =\sum_{s_{1}, s_{2}, m_{1}, m_{2}} \mid \int d \vec{R}_{12} e^{i \vec{P}_{12} \cdot \vec{R}_{12}} \bar{u}\left(\vec{k}_{1}, s_{1}\right) \psi_{n_{1} \kappa_{1} m_{1}}\left(\vec{R}_{12}\right) \\
& \quad \times\left.\bar{u}\left(\vec{k}_{2}, s_{2}\right) \psi_{n_{2} \kappa_{2} m_{2}}\left(\vec{R}_{12}\right) \mathcal{F}_{\mathrm{FSI}}\left(\vec{R}_{12}, \vec{R}_{12}\right)\right|^{2}
\end{aligned}
$$

Here, $u(\vec{k}, s)$ are positive-energy Dirac spinors and $\psi_{n \kappa m}$ are relativistic mean-field wave functions [44] with quantum numbers $(n, j=|\kappa| / 2, m)$. We neglect the projections on the lower components of the plane-wave Dirac spinors. The FSIs of the ejected pair with the remaining $A-2$ spectators, encoded in $\mathcal{F}_{\mathrm{FSI}}$, can be computed in a relativistic multiplescattering Glauber approximation (RMSGA) [45,46]. As the c.m. momentum is conserved in interactions among the two ejected nucleons, we discard those. This approximation does not affect the shape of $F_{n_{1} \kappa_{1}, n_{2} \kappa_{2}}^{D}\left(\vec{P}_{12}\right)$.

We include FSIs for the JLab data-mining kinematics considered in Sec. IV. We have computed the distorted c.m. momentum distribution of Eq. (45) for the kinematics

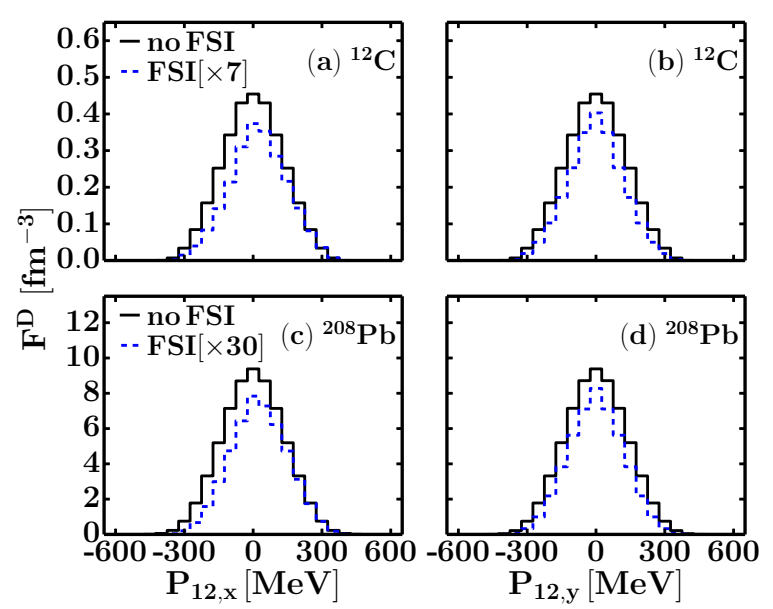

FIG. 10. (Color online) The two-body c.m. momentum distribution for ${ }^{12} \mathrm{C}\left(e, e^{\prime} p p\right)$ (top) and ${ }^{208} \mathrm{~Pb}\left(e, e^{\prime} p p\right)$ (bottom) with (RMSGA) and without (no-FSI) inclusion of FSIs. We consider the kinematics $|\vec{q}|=1.4 \mathrm{GeV},\left|\vec{p}_{1}\right|=0.82|\vec{q}|$, and $\theta_{\vec{p}_{1}, \vec{q}}=10^{\circ}$. The FSI results have been multiplied by a factor of 7 for ${ }^{12} \mathrm{C}\left(e, e^{\prime} p p\right)$ and by a factor of 30 for ${ }^{208} \mathrm{~Pb}\left(e, e^{\prime} p p\right)$. 


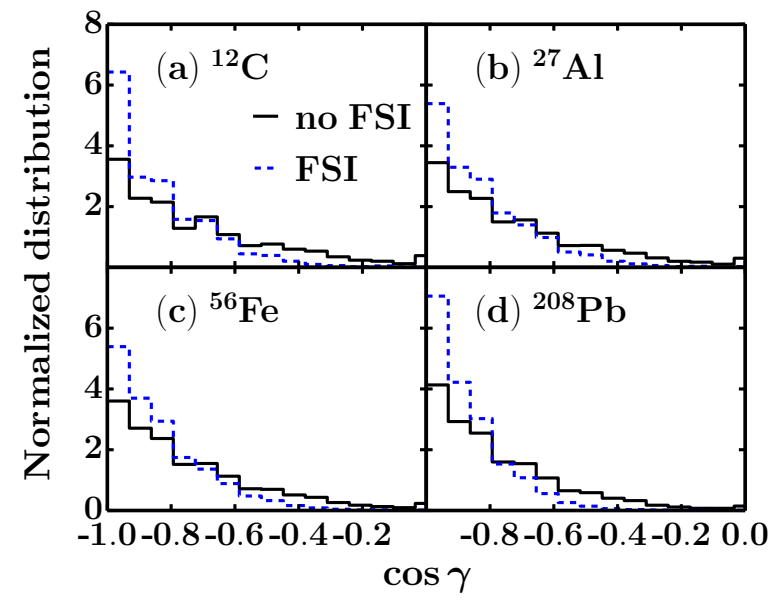

FIG. 11. (Color online) The normalized opening angle distributions for $\mathrm{A}\left(e, e^{\prime} p p\right)$ for ${ }^{12} \mathrm{C},{ }^{27} \mathrm{Al},{ }^{56} \mathrm{Fe}$, and ${ }^{208} \mathrm{~Pb}$ in the kinematics of Fig. 10.

that yields the most events in the simulations of Sec. IV: $|\vec{q}|=1.4 \mathrm{GeV},\left|\vec{p}_{1}\right|=0.82|\vec{q}|, \theta_{\vec{p}_{1}, \vec{q}}=10^{\circ}$. As in Sec. IV, $\vec{k}_{1}$ lies along the $z$ axis and the $\vec{q}$ is located in the $x z$ plane. The results of the FSI calculations are summarized in Figs. 10 and 11 .

In Fig. 10 we compare the RMSGA c.m. momentum distributions $F^{D}\left(\vec{P}_{12, x}\right)=\sum_{n_{1} \kappa_{1}, n_{2} \kappa_{2}} F_{n_{1} \kappa_{1}, n_{2} \kappa_{2}}^{D}\left(\vec{P}_{12, x}\right)$ and $F^{D}\left(\vec{P}_{12, y}\right)$ with their respective plane-wave (no-FSI) limit. First, the FSIs are responsible for a substantial reduction of the cross sections: a factor of about 7 for carbon and about 30 in lead. The effects of FSIs on the shape of $F^{D}\left(\vec{P}_{12}\right)$, however, are rather modest. Gaussian fits to the $F^{D}\left(\vec{P}_{12, i=x, y}\right)$ result in widths which are less than $10 \%$ smaller than in the plane-wave limit. The effects of FSIs on the shape of the c.m. distributions in Fig. 10 can be qualitatively understood considering that the nucleons undergoing FSIs are slowed down on average: $\left(\vec{p}_{1}, \vec{p}_{2}\right) \stackrel{\text { FSI }}{\longrightarrow} \zeta\left(\vec{p}_{1}, \vec{p}_{2}\right)$ with $0<\zeta \leqslant 1$. It is straightforward to show that for the adopted conventions this results in $P_{12, x} \rightarrow \zeta P_{12, x}-(1-\zeta) p_{1, x}$, and $P_{12, y} \rightarrow \zeta P_{12, y}$. This explains the observed contraction and shift to the right in the $P_{12, x}$ distribution, and the contraction of the $P_{12, y}$ distributions.

The effect of FSIs on the shape of the normalized opening angle distributions is studied in Fig. 11 for four target nuclei. It is clear that they become even more forwardly peaked after including FSIs.

\section{SUMMARY}

Summarizing, we have shown that in the plane-wave limit the factorization function for the exclusive SRCdriven $A\left(e, e^{\prime} p N\right)$ reaction is the conditional c.m. distribution $P_{2}\left(P_{12} \mid n l=00\right)$ for $p N$ pairs in a nodeless relative state with a vanishing orbital momentum. We have illustrated that in a two-body cluster expansion the correlated part of the momentum distribution originates mainly from correlation operators acting on IPM pairs with $(n l=00)$ quantum numbers, supporting the assumptions underlying the proposed factorization of the $A\left(e, e^{\prime} p N\right)$ reaction. Numerical calculations indicate that the $P_{2}\left(P_{12} \mid n l=00\right)$ has a wider distribution than the unconditional $P_{2}\left(P_{12}\right)$ one. An important implication of the proposed factorization is that the mass dependence of the $A\left(e, e^{\prime} p p\right)$ and $A\left(e, e^{\prime} p n\right)$ cross section is predicted to be much softer than $\frac{Z(Z-1)}{2}$ and $N Z$, respectively.

We have examined the robustness of the proposed factorization of the two-nucleon knockout cross sections against kinematic cuts and FSIs. Both mechanisms modestly affect the shape of the c.m. distributions which leads us to conclude that they can be accessed in $A\left(e, e^{\prime} p N\right)$ measurements. The FSIs bring about a mass-dependent reduction of the cross sections which is of the order of 10 for carbon and 30 for lead.

\section{ACKNOWLEDGMENTS}

The authors wish to thank Or Hen, Eli Piasetzky, and Larry Weinstein for stimulating discussions and suggestions. This work is supported by the Research Foundation Flanders (FWO-Flanders) and by the Interuniversity Attraction Poles Programme P7/12 initiated by the Belgian Science Policy Office. The computational resources (Stevin Supercomputer Infrastructure) and services used in this work were provided by Ghent University, the Hercules Foundation, and the Flemish Government.
[1] A. Tang, J. W. Watson, J. Aclander, J. Alster, G. Asryan, Y. Averichev, D. Barton, V. Baturin, N. Bukhtoyarova, A. Carroll et al., Phys. Rev. Lett. 90, 042301 (2003).

[2] R. A. Niyazov, L. B. Weinstein, G. Adams, P. Ambrozewicz, E. Anciant, M. Anghinolfi, B. Asavapibhop, G. Asryan, G. Audit, T. Auger et al. (CLAS Collaboration), Phys. Rev. Lett. 92, 052303 (2004).

[3] R. Shneor, P. Monaghan, R. Subedi, B. D. Anderson, K. Aniol, J. Annand, J. Arrington, H. Benaoum, F. Benmokhtar, P. Bertin et al. (Jefferson Lab Hall A Collaboration), Phys. Rev. Lett. 99, 072501 (2007).

[4] R. Subedi, R. Shneor, P. Monaghan, B. Anderson, K. Aniol, J. Annand, J. Arrington, H. Benaoum, F. Benmokhtar, W. Boeglin et al., Science 320, 1476 (2008).
[5] K. S. Egiyan, N. Dashyan, M. Sargsian, S. Stepanyan, L. B. Weinstein, G. Adams, P. Ambrozewicz, E. Anciant, M. Anghinolfi, B. Asavapibhop et al. (CLAS Collaboration), Phys. Rev. C 68, 014313 (2003).

[6] K. S. Egiyan, N. B. Dashyan, M. M. Sargsian, M. I. Strikman, L. B. Weinstein, G. Adams, P. Ambrozewicz, M. Anghinolfi, B. Asavapibhop, G. Asryan et al. (CLAS Collaboration), Phys. Rev. Lett. 96, 082501 (2006).

[7] N. Fomin, J. Arrington, R. Asaturyan, F. Benmokhtar, W. Boeglin, P. Bosted, A. Bruell, M. H. S. Bukhari, M. E. Christy, E. Chudakov et al., Phys. Rev. Lett. 108, 092502 (2012).

[8] R. Schiavilla, R. B. Wiringa, S. C. Pieper, and J. Carlson, Phys. Rev. Lett. 98, 132501 (2007). 
[9] R. B. Wiringa, R. Schiavilla, S. C. Pieper, and J. Carlson, Phys. Rev. C 78, 021001(R) (2008).

[10] H. Feldmeier, W. Horiuchi, T. Neff, and Y. Suzuki, Phys. Rev. C 84, 054003 (2011).

[11] R. Wiringa, R. Schiavilla, S. C. Pieper, and J. Carlson (2013), arXiv:1309.3794.

[12] M. Alvioli, C. Ciofi degli Atti, and H. Morita, Phys. Rev. Lett. 100, 162503 (2008).

[13] M. Alvioli, C. Ciofi degli Atti, L. P. Kaptari, C. B. Mezzetti, H. Morita, and S. Scopetta, Phys. Rev. C 85, 021001(R) (2012).

[14] M. Alvioli, C. Ciofi degli Atti, L. P. Kaptari, C. B. Mezzetti, and H. Morita, Phys. Rev. C 87, 034603 (2013).

[15] F. Arias de Saavedra, C. Bisconti, G. Co', and A. Fabrocini, Phys. Rep. 450, 1 (2007).

[16] C. Bisconti, F. A. d. Saavedra, and G. Co', Phys. Rev. C 75, 054302 (2007).

[17] S. K. Bogner and D. Roscher, Phys. Rev. C 86, 064304 (2012).

[18] J. Arrington, D. Higinbotham, G. Rosner, and M. Sargsian, Prog. Part. Nucl. Phys. 67, 898 (2012).

[19] L. Frankfurt, M. Sargsian, and M. Strikman, Int. J. Mod. Phys. A 23, 2991 (2008).

[20] M. Vanhalst, W. Cosyn, and J. Ryckebusch, Phys. Rev. C 84, 031302(R) (2011).

[21] M. Vanhalst, J. Ryckebusch, and W. Cosyn, Phys. Rev. C 86, 044619 (2012).

[22] M. Vanhalst, J. Ryckebusch, and W. Cosyn (2012), arXiv:1210.6175.

[23] W. Cosyn, M. Vanhalst, and J. Ryckebusch (2013), arXiv:1308.5583.

[24] J. Arrington, A. Daniel, D. Day, N. Fomin, D. Gaskell, and P. Solvignon, Phys. Rev. C 86, 065204 (2012).

[25] O. Benhar, Phys. Rev. C 87, 024606 (2013).

[26] R. Starink, M. van Batenburg, E. Cisbani, W. Dickhoff, S. Frullani, F. Garibaldi, C. Giusti, D. Groep, P. Heimberg, W. Hesselink et al., Phys. Lett. B 474, 33 (2000).

[27] M. Moshinsky and Y. Smirnov, The Harmonic Oscillator in Modern Physics (Harwood Academic Publishers, Amsterdam, 1996).
[28] S. C. Pieper, R. B. Wiringa, and V. R. Pandharipande, Phys. Rev. C 46, 1741 (1992).

[29] J. Engel, J. Carlson, and R. B. Wiringa, Phys. Rev. C 83, 034317 (2011).

[30] R. Roth, T. Neff, and H. Feldmeier, Prog. Part. Nucl. Phys. 65, 50 (2010).

[31] S. Janssen, J. Ryckebusch, W. Van Nespen, and D. Debruyne, Nucl. Phys. A 672, 285 (2000).

[32] J. Ryckebusch, V. Van der Sluys, K. Heyde, H. Holvoet, W. Van Nespen, M. Waroquier, and M. Vanderhaegen, Nucl. Phys. A 624, 581 (1997).

[33] C. Gearheart, Ph.D. thesis, Washington University, St. Louis, 1994.

[34] J. Caballero, T. Donnelly, E. Moya de Guerra, and J. Udias, Nucl. Phys. A 632, 323 (1998).

[35] L. L. Frankfurt and M. I. Strikman, Phys. Rep. 160, 235 (1988).

[36] J. Ryckebusch, Phys. Lett. B 383, 1 (1996).

[37] K. I. Blomqvist et al., Phys. Lett. B 421, 71 (1998).

[38] C. Barbieri, C. Giusti, F. D. Pacati, and W. H. Dickhoff, Phys. Rev. C 70, 014606 (2004).

[39] J. Ryckebusch and W. Van Nespen, Eur. Phys. J. A 20, 435 (2004).

[40] D. Middleton, J. Annand, C. Barbieri, C. Giusti, P. Grabmayr et al., Eur. Phys. J. A 43, 137 (2010).

[41] L. Weinstein, S. Kuhn et al., "Short distance structure of nuclei: Mining the wealth of existing Jefferson Laboratory data," DOE Grant No. DE-SC0006801 (2009).

[42] O. Hen et al. (CLAS Collaboration), Phys. Lett. B 722, 63 (2013).

[43] W. Cosyn and J. Ryckebusch, Phys. Rev. C 80, 011602(R) (2009)

[44] R. J. Furnstahl, B. D. Serot, and H.-B. Tang, Nucl. Phys. A 615, 441 (1997).

[45] J. Ryckebusch, D. Debruyne, P. Lava, S. Janssen, B. Van Overmeire, and T. Van Cauteren, Nucl. Phys. A 728, 226 (2003).

[46] W. Cosyn and J. Ryckebusch, Phys. Rev. C 87, 064608 (2013). 